\title{
AN ADAPTATION OF THE THERMAL CONDUCTIVITY METHOD TO THE ANALYSIS OF RESPIRATORY GASES ${ }^{1}$
}

By PAUL G. LEDIG AND RICHARD S. LYMAN

(From the United States Bureau of Standards and the School of Medicine and Dentistry, University of Rochester)

(Received for publication May 4, 1927)

A simple and rapid method of analyzing respired air for carbon dioxide and oxygen is still needed in medicine. In investigations on the expenditure of energy in muscular exercise, many samples of expired gas must be analyzed daily. The number of such analyses has been further increased by the widespread adoption of routine clinical determinations of metabolic rates involving the respiratory exchange. An improvement in the technic of obtaining such data might also be applied to studies of the composition of alveolar air and possibly to the estimation of lung volumes. Accordingly it has seemed worth while to apply to the requirements of the medical profession the method of gas analysis by thermal conductivity which has proved of value along different lines to chemists and engineers.

Although gas analysis by thermal conductivity was shown to be feasible as early as about 1880 , it was not developed in medicine until recently and then only for the determination of carbon dioxide. In 1922, A. V. Hill (1) reported in the Proceedings of the Physiological Society the possibilities of the "katharometer" for the rapid determination of carbon dioxide in expired air. In 1926 Rabinowitch and Bazin (2) gave a more detailed account of this apparatus as produced by the Cambridge Instrument Company, and described its application for obtaining basal metabolic rates. They used a "katharometer" in conjunction with a gas meter, getting in that way the data from which they calculated the metabolic rate by the method previously described by King (3). A similar apparatus, manufactured by

${ }^{1}$ Published by permission of Director, Bureau of Standards, Department of Commerce, Washington, D. C. 
Siemens and Halske of Berlin, was used by Knipping (4) for the determination of carbon dioxide in alveolar airs.

The "katharometer" measures the change of resistance in an unbalanced Wheatstone bridge by the deflection of the needle of a millivoltmeter. In the apparatus developed by the United States Bureau of Standards, ${ }^{2}$ which is used in the present investigation, the resistances in a Wheatstone bridge are balanced by using a slide wire to bring a galvanometer to zero deflection. This type of thermal conductivity apparatus has been described by Palmer and Weaver (5), and so only a brief description will be given here.

Two fine platinum wires of equal resistance, enclosed in tubes 1 $\mathrm{cm}$. in diameter, comprise two arms of the Wheatstone bridge, and are heated by an electric current. When the wires are surrounded by gas of the same composition, heat is conducted from them through the gas at the same rate, maintaining their electrical resistances constant and equal. Any change in the composition of the gas around one wire is attended by a proportional change in the quantity of heat conducted by the gas from the wire and, therefore, by a corresponding change in its temperature and electrical resistance. If the composition of the gas around the second wire remains constant, the difference between two settings of the slide wire required to bring the bridge to balance, is a measure of the change in the composition of the gas which surrounds the first wire. The significance of the readings can be determined only when the instrument has been calibrated for variations in the amount of one constituent in a gas mixture, the other constituents of which remain substantially constant; the instrument cannot be used for qualitative analysis in any case.

\section{DESCRIPTION}

Figure 1 gives a diagram of the electrical circuit used. Current is supplied by a storage battery. The voltage of the circuit is kept constant by adjusting the rheostat, so that the galvanometer in the voltage-adjustment bridge is brought to zero reading. One arm of this bridge is a small lamp with a tungsten filament. The opposite arm

2 The authors are greatly indebted to E. R. Weaver of the Bureau of Standards' staff for his advice and encouragement throughout the difficulties attending the development of this instrument. 
is a lamp with a carbon filament, and the remaining arms are fixed resistances of manganin wire. The bridge functions by virtue of the fact that the electrical resistance of carbon decreases with rising tem-

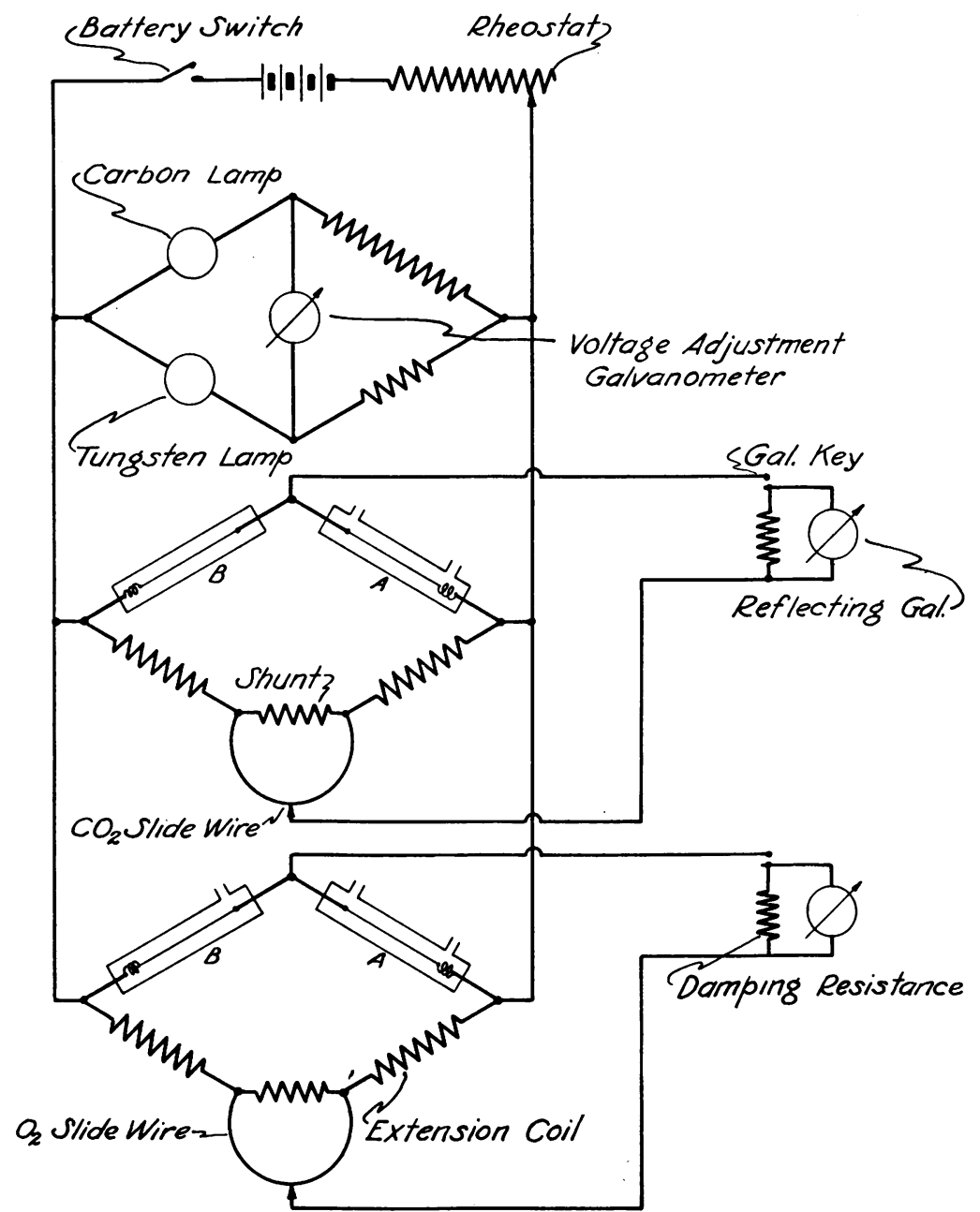

Fig. 1. Adaptation of Thermal Conductivity Method to Analysis of RESPIRATORY Gases

perature, while that of tungsten increases. A change of voltage will produce a corresponding change in the temperature of the lamp fila- 
ments and will unbalance the bridge. The voltage in the circuit can therefore be kept constant by maintaining the balance of the bridge, as indicated by a moderately sensitive galvanometer.

In the other two bridges, each platinum wire forming one of the arms is enclosed in a cylindrical brass cell from which the wire is properly insulated. Cell $\mathrm{A}$ in each bridge is equipped with two metal tubes, through which passes the gas to be analyzed, thus serving

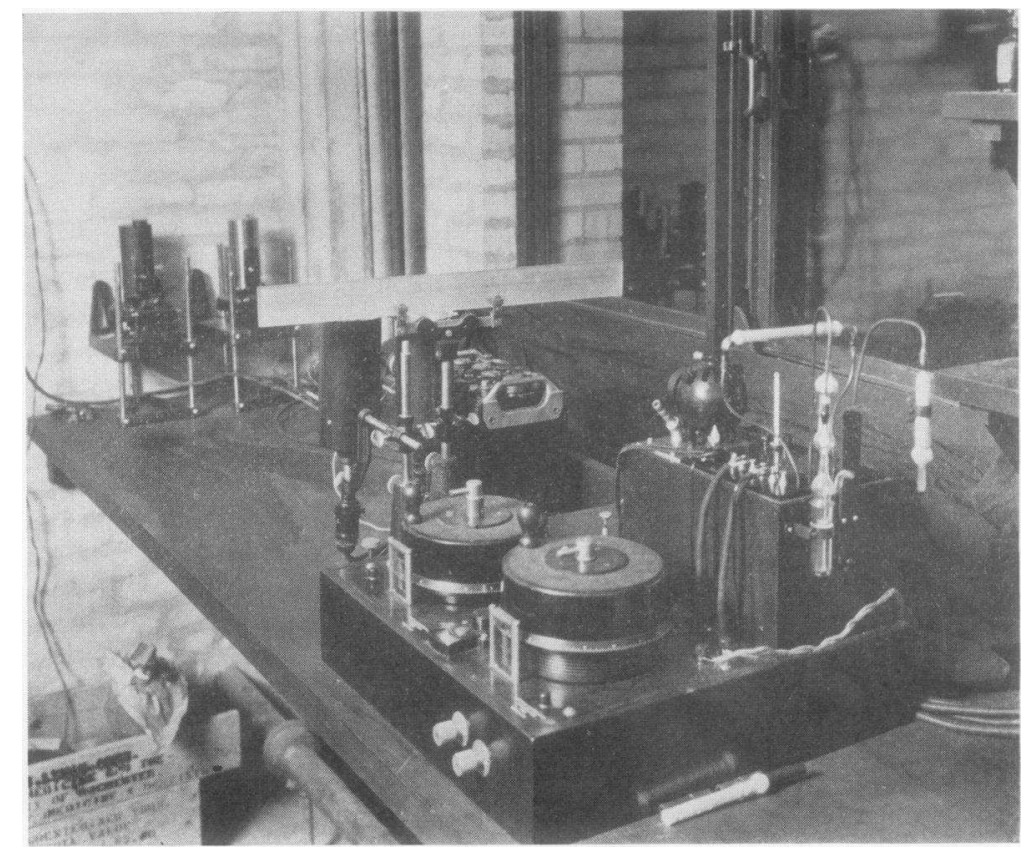

FIG. 2

as the "analysis cell." Cell B is filled with gas of a constant composition (air in this case) which serves as a standard of comparison. In the carbon dioxide bridge, this cell is sealed off. In the oxygen bridge, where the sensitivity must be much greater, it is open to the room air through a drying tube, in order to compensate for the effect of variations in barometric pressure. The other two arms of each analysis-bridge consist of the two end-coils (28.95 ohms each) and a shunted Kohlrausch slide: wire. The sensitivity of the bridge is de- 
termined by the resistance of the shunt across the ends of the slide wire. In order to make the bridge for oxygen analysis sufficiently sensitive, the resistance of its shunt is extremely low (less than onetenth of that across the slide wire on the bridge for carbon dioxide analysis). Highly sensitive mirror galvanometers are used to indicate when these bridges are balanced.

The thermal conductivity cells are kept at a constant temperature of $40^{\circ} \mathrm{C}$. by immersion in a small oil bath electrically stirred and heated. The temperature is controlled by means of a mercury thermo-regulator. To further decrease the effect of variations of temperature, the cells are enclosed in copper jackets $(3.5 \mathrm{~cm}$. square and slightly larger than the cells) which are then filled with fine shot. This volume of metal around the cells provides sufficient heat capacity to virtually eliminate all fluctuations in temperature. The influence of temperature on the bridge for oxygen analysis is further minimized by keeping its end-coils in the oil bath. Connections from these coils to the ends of the slide wire are made of heavy copper wire. Figure 2 is a photograph of the apparatus as used in the laboratory.

\section{METHOD OF OPERATION}

It has been found that 200 to $250 \mathrm{cc}$. of gas will sufficiently sweep out the system and give a dependable reading with this apparatus. The gas passes first through a drying tube into the analysis-cell of the carbon dioxide bridge, then through reagents which remove carbon dioxide and the water vapor which is produced during the absorption of carbon dioxide, and continues through the analysiscell of the oxygen bridge. A small bubbling tube is connected to its outlet to indicate the rate of gas flow. It is possible to pass a sample through in one minute. Higher rates than this do not give sufficient contact with the reagents to completely remove water and carbon dioxide. The sampler is then disconnected to insure atmospheric pressure in the cells, leaving them filled with the gas sample, which remains constant for some time, since diffusion to the atmosphere takes place very slowly. The slide wires of both bridges are adjusted to bring the deflections of the galvanometers to zero, and the settings are read directly from the slide-wire scales.

The apparatus is extremely sensitive to slight changes in electrical resistance. Fluctuations of room temperature and of atmospheric pressure, as well as other less important factors, produce variations in the readings obtained from the same gas at different times. These sources of inaccuracy are too great to be ignored, but they are compensated for if room air, from which carbon dioxide and water vapor have been removed, is swept through before each analysis. Readings on 


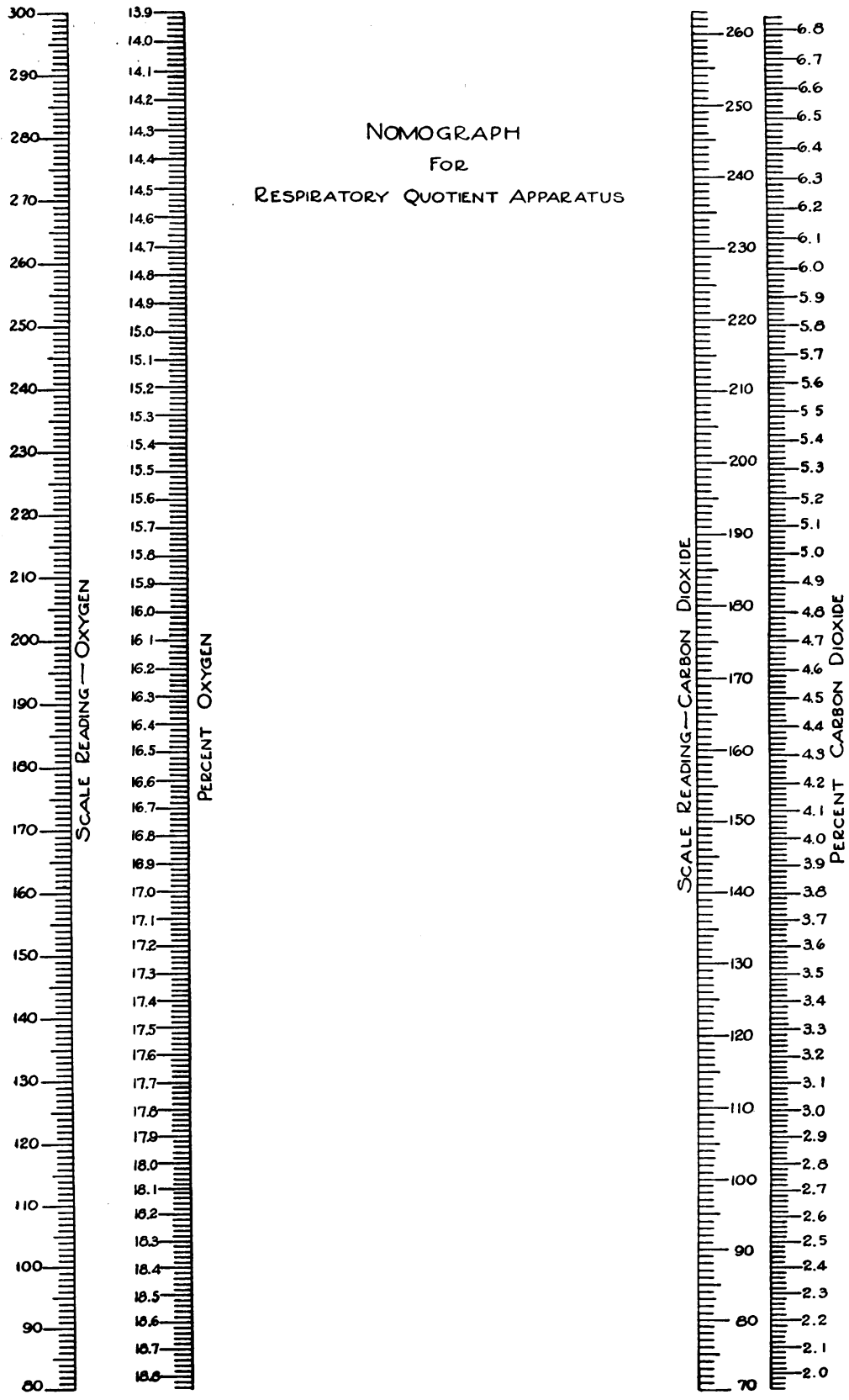

FIG. 3 
both bridges which are taken on this air serve as "base-line" readings. Differences between these readings and those obtained from the sample analyzed are the "scalereadings" used in determining the results of the analysis.

Two solid reagents of relatively recent introduction are used. For removing water-vapor Dehydrite (magnesium perchlorate trihydrate) (6) has been found the most satisfactory. Ascarite (sodium hydroxide deposited on asbestos fiber) has been found the best for removing carbon dioxide. Soda lime is unsatisfactory because it does not completely absorb carbon dioxide from a thoroughly dried gas.

An empirical calibration of each thermal conductivity apparatus is necessary because there is no direct method of determining what the changes of electrical resistance indicated may mean in terms of gas composition. It was necessary in this case to completely calibrate the apparatus against analyses of respired air made with the Haldane apparatus. Scale readings were plotted against percentages obtained by the Haldane method for both oxygen and carbon dioxide. The curves so produced covered the useful range of the apparatus for physiological purposes, which is about two to seven per cent of carbon dioxide and fourteen to eighteen and a half per cent of oxygen. From these curves a nomograph was constructed (fig. 3). A straight edge laid across the scale readings as they appear on it gives directly the percentages of carbon dioxide and of oxygen. On the oxygen side of the graph a correction is incorporated which allows for the loss of gas volume on absorption of carbon dioxide before the oxygen reading is made. In addition, a correction is made in the carbon dioxide scale to provide for slight changes in thermal conductivity in the carbon dioxide cell as the proportions of oxygen and nitrogen vary in the gas sample.

\section{DISCUSSION}

The adoption by the medical profession of technical improvements developed in the field of physics or chemistry may be considered justified if they increase the accuracy, simplify the manipulation, decrease the cost, or shorten the time involved in obtaining the data desired. Methods of gas analysis with a Haldane or Carpenter apparatus have given results of sufficient precision for the physiological applications in which they are commonly used. The time required to obtain duplicate analyses has however been their chief drawback. It is the rapidity with which the apparatus described in this paper operates that constitutes its main contribution to physiology and medicine-allowing more samples to be analyzed by one person than formerly was possible, or else releasing investigators for a closer study of results. The actual time taken for duplicate analyses of gas samples is short. One sample after another may be analyzed at a rate of about 
TABLE 1

Analyses made by the thermal conductivity method and by the Haldane method are compared. Analyses of respired gases made by the thermal conductivity method are used to determine respiratory quotients during a nine day dietary experiment

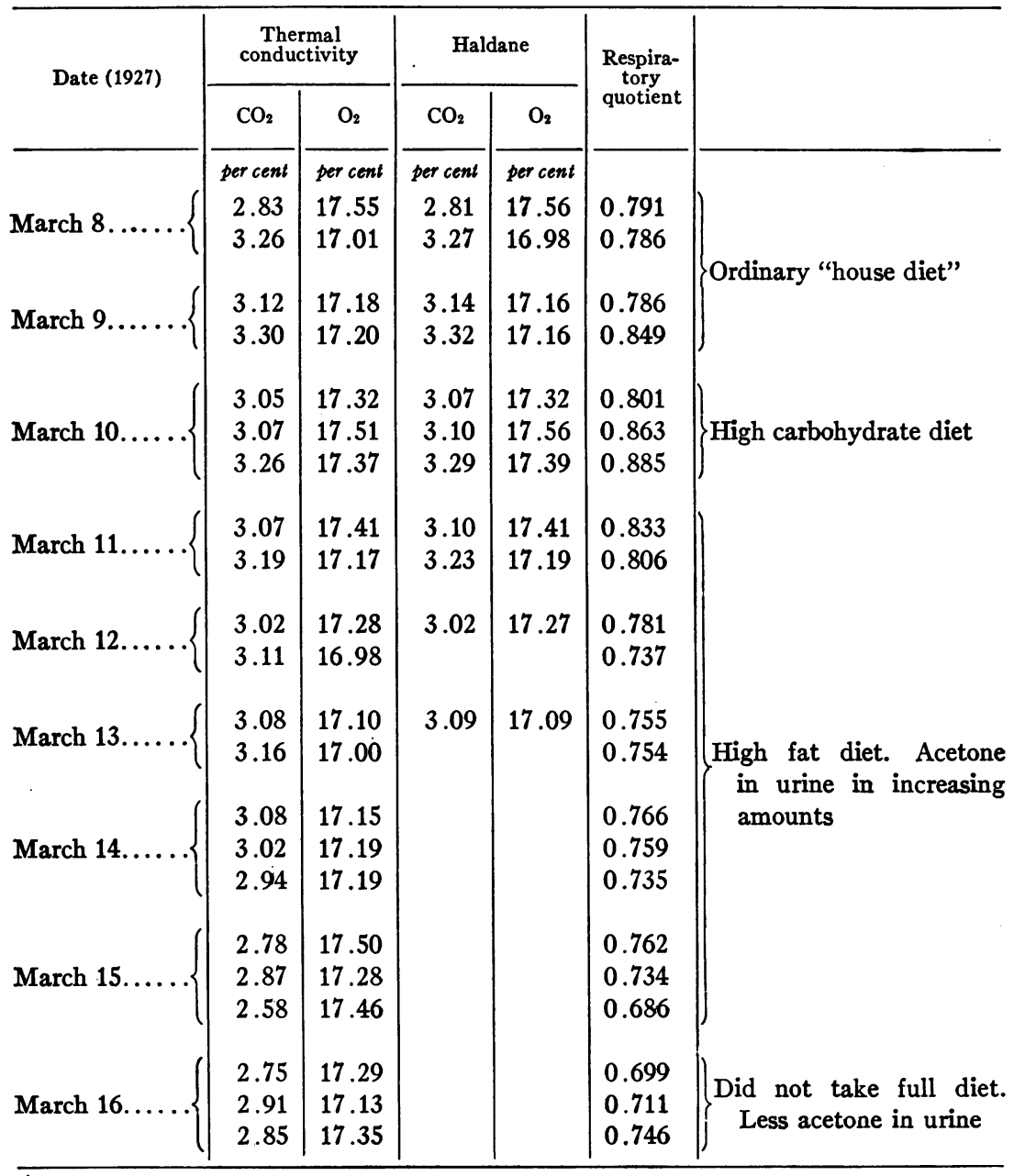

The first set of figures on each day was obtained under the post-absorptive conditions of routine basal metabolic rate determinations. The last set was taken on each day in the mid-afternoon. When three sets occur, the second shows results obtained about two hours after breakfast. 
one every five to seven minutes, alternating, or at least being interspersed with, "base-line" readings as described in the procedure.

An automatic recorder for making a continuous curve to indicate the fluctuations in the thermal conductivity of a stream of gas flowing through such an apparatus would be desirable. A recorder has, in fact, been used with "katharometers" for recording carbon dioxide concentrations only. But it is impossible to apply it to the determination of oxygen with sufficient accuracy to be of value, because recording instruments of the necessary sensitivity have not yet been produced.

The actual manipulation is simple. There are only a few operations which enter into the process of making an analysis: (1) introducing the sample, (2) bringing the galvanometer-needle on the voltage adjustment bridge to zero, (3) adjusting the galvanometer reflections in the analysis-bridges by means of the slide wires, (4) reading the slide wire settings, (5) getting the differences between the baseline and the analysis readings by substraction, and (6) reading the percentages of carbon dioxide and oxygen directly from the nomograph. No further calculations are necessary in using this apparatus. This eliminates a possibility of error which is always present in making analyses with the Haldane method. With regard to keeping the apparatus in operating condition, one need only attend to charging the battery at intervals and to changing the reagents in the drying tubes occasionally. The galvanometers may require occasional adjustment and it is necessary to see that the stirring motor and temperature control of the oil bath are operating satisfactorily. All of these details take less time than is necessary to keep one Haldane analyzer in good condition.

The accuracy obtainable is satisfactory for all practical uses involving the respiratory exchange. The first criterion of the accuracy of the method is its ability to reproduce results. A gas mixture was made up at high pressure in a small steel cylinder. Twenty-seven readings made on the gas from this cylinder over a period of three weeks, checked each other with a maximum deviation from the mean of 0.02 per cent for oxygen. Carbon dioxide readings checked each other within 0.01 per cent. In making the nomograph the calibration of this apparatus was found to fit the analyses made by the 
Haldane method with a maximum difference of no more than 0.05 per cent for both gases. This calibration has since been put to test by a series of metabolic rates carried out on a patient before and after being put on a high-fat diet, as shown in table 1. All check analyses made by the Haldane method came within 0.05 per cent of each other and of those made by the thermal conductivity method. There is no reason to assume that the failure of the two apparatuses to check each other perfectly was caused by errors of the thermal conductivity apparatus. It is equally possible that the errors may have been made in individual cases by the Haldane method.

The advantages of the new method of analyzing respiratory gases are accompanied by a few definite limitations. Of these, probably the most serious is the cost of the sensitive and specialized equipment. Only certain institutions will have occasion to purchase this type of apparatus, both because of its cost and because in them alone would the demand for air analyses warrant its use. A further disadvantage is the necessity for a complete recalibration in case of damage to one or more of the thermal conductivity cells by accident or excessive current. This might put the apparatus out of use for some time. The size of the gas sample needed to sweep out the system may in some cases also limit the field to which this apparatus can be applied. Although this difficulty has not been investigated, it is barely possible that in certain pathological conditions the expired air may contain gases or-vapors (such as acetone in diabetes) which would not affect the analysis made by the Haldane apparatus, but which might slightly shift the thermal conductivity readings. And finally, in case of repairs, the services of a skilled worker familiar with electrical measurements would be essential.

\section{SUMMARY}

1. An apparatus has been developed for the analysis by thermal conductivity of both carbon dioxide and oxygen in respiratory gases.

2. The principles of construction and method of operation are described.

3. Its potential value to medical science depends upon: (a) Saving of time; duplicate analyses may be completed in ten minutes. (b) simplicity of operation; (c) virtual elimination of calculation; (d) requisite accuracy. 
4. The only limitations which appear significant are: (a) Cost of construction; (b) necessity for complete recalibration in case of accident.

\section{BIBLIOGRAPHY}

1. Reported by Daynes, H. A., Proc. Roy. Soc., Series A, 1920, xcvii, 273. The Theory of the Katharometer.

2. Rabinowitch, I. M., and Bazin, E. V., Can. Med. Assoc. Jour., 1926, xvi, 638. A Simple and Accurate Method of Determining Basal Metabolic Rates. An Electrometric (Katharometer) Procedure.

3. King, John T., Jr., Johns Hopkins Hosp. Bull., 1921, xxxii, 277. Determination of the Basal Metabolism from the Carbon-Dioxide Elimination.

4. Knipping, H. W., Zeit. Phys. Chem., 1924, cxli, 1. Uber die Bestimmung der Kohlensäurespannung in der Alveolarluft.

5. Palmer, P. E., and Weaver, E. R., Technologic Papers of the Bureau of Standards, 1924, xviii, 35. Thermal-Conductivity Method for the Analysis of Gases.

6. Willard, H. H., and Smith, G. F., Jour. Am. Chem. Soc., 1922, xliv, 2255. The Preparation and Properties of Magnesium Perchlorate and its Use as a Drying Agent. See also Ind. Eng. Chem., 1924, xvi, 20. 